\title{
Trapping, self-trapping and the polaron family
}

\author{
A M Stoneham ${ }^{1}$, J Gavartin ${ }^{1}$, A L Shluger ${ }^{1}$, A V Kimmel ${ }^{1}$, \\ D Muñoz Ramo ${ }^{1}$, H M Rønnow ${ }^{2}$, G Aeppli $^{1}$ and C Renner ${ }^{1}$ \\ ${ }^{1}$ London Centre for Nanotechnology and Department of Physics and Astronomy, \\ University College London, Gower Street, London WC1E 6BT, UK \\ ${ }^{2}$ Laboratory for Neutron Scattering ETH Zürich and Paul Scherrer Institut, 5232 Villigen, \\ Switzerland
}

Received 29 September 2006, in final form 7 November 2006

Published 30 May 2007

Online at stacks.iop.org/JPhysCM/19/255208

\begin{abstract}
The earliest ideas of the polaron recognized that the coupling of an electron to ionic vibrations would affect its apparent mass and could effectively immobilize the carrier (self-trapping). We discuss how these basic ideas have been generalized to recognize new materials and new phenomena. First, there is an interplay between self-trapping and trapping associated with defects or with fluctuations in an amorphous solid. In high dielectric constant oxides, like $\mathrm{HfO}_{2}$, this leads to oxygen vacancies having as many as five charge states. In colossal magnetoresistance manganites, this interplay makes possible the scanning tunnelling microscopy (STM) observation of polarons. Second, excitons can self-trap and, by doing so, localize energy in ways that can modify the material properties. Third, new materials introduce new features, with polaron-related ideas emerging for uranium dioxide, gate dielectric oxides, Jahn-Teller systems, semiconducting polymers and biological systems. The phonon modes that initiate self-trapping can be quite different from the longitudinal optic modes usually assumed to dominate. Fourth, there are new phenomena, like possible magnetism in simple oxides, or with the evolution of short-lived polarons, like muons or excitons. The central idea remains that of a particle whose properties are modified by polarizing or deforming its host solid, sometimes profoundly. However, some of the simpler standard assumptions can give a limited, indeed misleading, description of real systems, with qualitative inconsistencies. We discuss representative cases for which theory and experiment can be compared in detail.
\end{abstract}

(Some figures in this article are in colour only in the electronic version)

\section{The polaron concept: how electron-lattice coupling transforms electron behaviour}

For a free carrier in a perfect crystal, its dynamical behaviour under applied fields is determined partly by electronic structure and partly by the way the carrier deforms or polarizes its host. 
In many systems, a mere modification of the effective mass encompasses quite a few of the interesting effects. When translational symmetry is present, such simplicity is not unexpected, although it is perhaps surprising that this superficial simplicity includes many-body effects in effective mass theory of shallow defects in semiconductors. There are natural generalizations of this standard polaron picture to take account of inertia associated with other, non-vibrational, degrees of freedom, e.g., magnetic, polarization of conduction electrons in a nearby media, and so on. Our present paper is concerned primarily with localized carriers for which polaron ideas are crucial, especially self-trapping, first predicted by Landau. We shall build on several previous reviews (Stoneham 1989, Shluger and Stoneham 1993). Self-trapping of excitons is important, not least because the exciton self-trapping causes energy localization that can enable defect processes; there are both fuller references and an extensive comparison with a rather wide range of experiments in Itoh and Stoneham 2001. Other aspects are reviewed by Alexandrov and Mott (1995). We shall begin with an analysis of the self-trapping of carriers and of excitons in otherwise perfect crystals, emphasizing the influence of polaron behaviour on significant practical issues. Next, we discuss the interplay of such intrinsic self-trapping with trapping and localization associated with defects or disorder. We then turn to polaron dynamics in various forms: in the self-trapping process, and in various coherent and incoherent motions, including oxide superconductors and colossal magnetoresistance oxides in which the JahnTeller effect plays a role. Finally, we turn to optical and magnetic properties of polarons, in which excited state issues are important. Our aim is to show some of the underlying coherence in the ideas and varied phenomena usually classed as polaron behaviour.

Landau's seminal paper (Landau 1933) made three key points. First, the immobilization of carriers (self-trapping) should occur for strong electron-lattice coupling. This prediction was right, verified in many experiments, and extended by Holstein (1959) and by Yamashita and Kurosawa (1958) in discussions of the incoherent diffusive motion of self-trapped carriers. Second, he argues that there should be an energy barrier to self-trapping. This is sometimes the case, e.g., for self-trapped excitons in alkali iodides, but is observed only rarely (Mott and Stoneham 1977, Shluger and Stoneham 1993). Third, he suggested that the F-centre in alkali halides might be an example of a self-trapped electron. This idea proved wrong, but the hole in alkali halides did self-trap, with properties similar to those predicted (see below).

Subsequently Fröhlich (1962), Feynman (1955), Pekar (1951), Toyozawa (1962), and others developed ideas of the dynamics of polarons that did not self-trap, but were describable by an effective mass. These cases lie outside our present discussion, concentrating on localized polarons. We focus on ideas that have been generalized substantially as real systems were understood, especially as the links became understood between self-trapping and the energy localization that drives material modifications. Such ideas include recognition of the inertia of some other degrees of freedom (vibrational, magnetic, etc) inhibiting electronic carrier motion between states that are related by translational symmetry. The carrier can exhibit new types of state that are self-trapped, distinct in defined ways. Trapping and self-trapping can, and often do, occur in combination. There may be an energy barrier between the self-trapped and non-self-trapped states. Electron-phonon coupling can lead to distinct regimes of electron motion, specifically coherent (with its own distinct forms) and incoherent. More subtle forms of electron-phonon coupling are sometimes also referred to as polaronic, e.g., the Ham effect (Ham 1965), when vibronic matrix elements are compared with purely electronic ones, or other cases where vibrational overlap factors emerge, e.g., Huang-Rhys theory for optical and non-radiative transitions (Huang and Rhys 1950, Stoneham 1975). When there is electronic degeneracy, it is unwise to assume that the simplest oscillator models still apply.

The idea of self-trapped electron states appears to violate symmetry rules. The self-trapped hole (Kanzig 1955; section 2.2 here) in alkali halides appears to violate both translational 
and local point symmetry and Bloch's theorem. The hole localized on one of the six nearestneighbour oxygens to an $\mathrm{Mg}$ vacancy in $\mathrm{MgO}$ (the $\mathrm{MgO} \mathrm{V}^{-}$centre) appears to violate point symmetry. The violation is apparent, rather than real: there is an equal probability of the carrier being at any one of the symmetry-related sites, and a single site will be identified only by experiments that have a characteristic timescale shorter than that for motion between equivalent sites. The situation can become more complex for excitons or muons that can self-trap but which have a finite lifetime.

Some important ideas can be deduced from scaling arguments showing the different importances of specific energy terms as the wavefunction scale length $L$ is varied from a reference value $L_{o}$. Emin and Holstein (1976) identify four classes of behaviour. In three dimensions, the kinetic energy scales as $+L^{-2}$. Coulomb terms (including deformationinduced polarization) scale as $-L^{-1}$; short-range terms, as from the deformation potential, scale as $-L^{-3}$; and various cross-terms scale as $-L^{-2}$. Polaron localization occurs when the energy gain from the electron-lattice coupling offsets increases in other energies, such as strain energy and kinetic energy. With only Coulomb and kinetic energy terms, there is a single minimum in energy as a function of $L$, and so there is just one class of polaron, the so-called large polaron. When there are only short-range and kinetic energy terms, the energy is lowest for zero $L$, which would be the small polaron if atomic structure were included, or for infinite $L$, i.e., a free carrier. When both short-range and Coulomb terms are present with the kinetic energy, there are both small-polaron and large-polaron forms; the large polaron may be metastable or unstable. These continuum pictures provide a useful framework of understanding the real systems that are inevitably more complicated.

A wealth of calculations include lattice structure, and examples are given in later sections. Some common polaron models oversimplify, and so have only descriptive, rather than predictive, power. Indeed, such models may fail, notably when the model Hamiltonian does not capture correctly the often delicate balance between the several energy contributions discussed above. Thus, many tight-binding models (including Hartree-Fock) fail to account properly for the kinetic energy increase with localization, and so predict charge trapping when they should not. In contrast, standard Kohn-Sham density functional methods inherently minimize the kinetic energy of an electron, and so fail to predict localization and self-trapping correctly, even in very well attested cases, such as hole trapping at Al impurities in silica (Laegsgaard and Stokbro 2001, Pacchioni et al 2001) or exciton self-trapping in $\mathrm{NaCl}$ (Gavartin et al 2003) to name a few. The simple one-frequency, linear-coupling, harmonic, model can be qualitatively incorrect (see below for the $\mathrm{MgO}: \mathrm{V}^{-}$centre). Yet even modest atomistic modelling methods can work well, such as methods based on the shell model and on the Mott-Littleton embedding approach (Stoneham 1989, Shluger and Stoneham 1993).

All simple models indicate that materials with very high dielectric constants should have strong coupling between electrons and ions, and so are likely to show significant polaron effects. Thus the so-called high- $k$ oxides being considered for future gate dielectric technologies should show evidence of polaronic behaviour. For this reason, we shall illustrate some of the wealth of such behaviour with results for $\mathrm{HfO}_{2}$ and closely related systems.

\section{The self-trapped polaron}

In this section, we sketch established results for free small-polaron systems. In part it is to indicate the wealth of validation of the basic ideas, both through experiment and theory, especially for the self-trapped hole in alkali and alkaline earth halides and for the self-trapped exciton in $\alpha$-quartz. But first it is important to point to the wide-ranging practical importance of charge localization and energy localization. Self-trapping has been observed for electrons, 
holes, excitons, and for muons and muonium. The phenomenon is of much more than scientific interest. The self-trapping of excitons, for example, leads to energy localization: significant amounts of energy associated with the excited state are concentrated onto a few atoms, and can enable reconstructions and atomic motions that underlie a wide range of methods for material modification (Itoh and Stoneham 2001).

\subsection{The serious small polaron: $\mathrm{UO}_{2}$ and reactor safety}

In safety studies of nuclear reactors, the thermal and mechanical behaviour of uranium dioxide at high temperatures is important. In $\mathrm{UO}_{2}$ both the electron (roughly $\mathrm{U}^{3+}$ ) and the hole (roughly $\mathrm{U}^{5+}$ ) form small polarons. At low temperatures, holes dominate in $\mathrm{UO}_{2+x}$. As first recognized by Devreese (1963), $\mathrm{UO}_{2}$ is an intrinsic polaronic semiconductor. In the temperature range relevant for modelling safety incident scenarios, the key thermal properties (specific heat, thermal conductivity) and even mechanical properties (stability against slumping) appear to be dominated by these small polarons. For a safety case, the credibility of the results (experimental and theoretical) is crucial: there is a need for understanding that can be trusted. As an example, careful measurements of the frequency-dependent dielectric function under various conditions of temperature and pressure provided a significant link between theory and technological data, partly because of the information given on the incoherent motion of self-trapped polaronic carriers (e.g., Hampton et al 1987).

The case of $\mathrm{UO}_{2}$, in which the electron small polaron and the hole small polaron can both be described with acceptable accuracy as changes in uranium ionic charge state, identify a link to many other systems. One must be cautious about assuming that carriers are localized on cations in transition metal oxides, even though the transition metal ion may be able to take several charge states. But the key energy terms are common to many cases: there is a Madelung energy, linear in the charge change $\delta Z$ from the perfect solid; there is a polarization term roughly proportional to $(\delta Z)^{2}$; and there are smaller crystal-field and other short-range terms. There is a similar balance of terms when one asks which are the stable charge states of dopants (say a transition metal ion at the $\mathrm{Mg}$ site in $\mathrm{MgO}$ (Stoneham and Sangster 1980)), providing a test of methods. It is the polarization term that often drives the localization of a carrier in oxides (Hayes and Stoneham 1985 p 35). Consider the carrier being spread over $N$ sites. Ignoring interference effects, defect forces $F / N$ drive deformation and polarization, being proportional to the fraction of charge at each site. The $N$ contributions each contribute relaxation energies $(F / N)^{2} / 2 K$, with $K$ the relevant force constant, so that the total stabilization energy for the charge is $N(F / N)^{2} / 2 K$, which is proportional to $1 / N$. For greatest stability, the carrier would be on a single site. The argument is simplistic as given, but full calculations confirm that concentrating the defect forces on a small number of atoms is usually favoured energetically. Whether or not self-trapping occurs depends on the relative values of this stabilization energy and the kinetic energy cost of carrier localization (see Shluger and Stoneham 1993).

\subsection{Halides: $V_{k}$ centres and tests of ideas}

In alkali and alkaline-earth halides, electrons do not self-trap: the conduction band is wide, and the localization energy is too high. However, holes self-trap, driven partly by polarization and partly by chemistry (two $\mathrm{Cl}^{-}$ions plus a hole can bond to form a molecular ion, $\mathrm{Cl}_{2}^{-}$). A 'molecule in a crystal' model works very well quantitatively (Tasker and Stoneham 1977). In such an approach, a full-scale molecular electronic structure calculation might be used for the halogen molecular ion, with this molecular ion embedded in a host crystal using a generalization of the methods pioneered by Mott and Littleton (1938). Ionic and electronic 


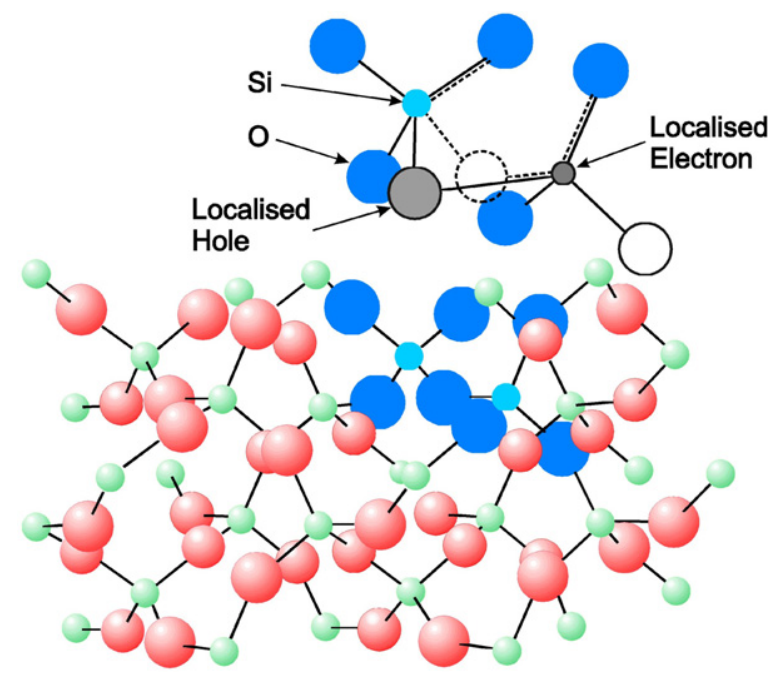

Figure 1. The self-trapped exciton in $\alpha$-quartz (after Itoh and Stoneham 2001), showing the localization of the electron and hole components, and the displacement of the oxygen from its normal site.

polarization must be treated at least at the shell model level: rigid ion models give relatively poor accuracy. Puchin et al (1993) and Shluger et al (1995) used a more elaborate embedding scheme and again found this 'molecule in a crystal' approach extremely successful. There are predictions of formation energies, optical transition energies, Arrhenius energies for the several different incoherent hopping transitions, the dipole tensor, the spin resonance hyperfine constants, and so on (Tasker and Stoneham 1977, Norgett and Stoneham 1973a, 1973b). Shluger et al (1992) have developed the theory for the $60^{\circ}$ reorientations of $V_{k}$ centres in alkali halides. Several points must be made. First, the formation energies are not measured directly, so theory can only confirm that the self-trapped small polaron is the stable form (Cade et al 1984). Second, the Arrhenius energy measured, $U$, is related to the temperature-dependent hopping rate $W(T)$ by $U=k T^{2} \mathrm{~d} / \mathrm{d} T[\ln \{W(T)\}]$, which comes from a fit to the slope of the standard Arrhenius plot of $\ln \{W(T)\}$ versus $1 / T$. As discussed by Norgett and Stoneham ((1973b), especially their figure 2) in the theory of Holstein (1959) and of Yamashita and Kurosawa (1958), this is not the same as their 'activation energy', since, even after allowing for the temperature-dependent prefactor, $U$ should be significantly smaller at the lower temperatures at which most experiments are done.

\subsection{Energy localization: the self-trapped exciton in $\alpha-q u a r t z$}

In quartz, i.e., crystalline $\mathrm{SiO}_{2}$, the electron and hole do not self-trap, although they localize very easily, e.g., at Ge impurities. However, the exciton does self-trap. As can be seen from figure 1, the self-trapped exciton has the electron primarily on a silicon, and the hole on an oxygen that has relaxed away from its normal site. It is no surprise that, in a fraction of cases, an oxygen vacancy and an oxygen interstitial can form, an example of material modification following electronic excitation and energy localization. In amorphous silicas, it is through processes like this that Bragg gratings are written in optical fibres. For self-trapped excitons, a large Stokes shift is often a symptom of self-trapping. Thus, in quartz, the exciton absorption energy is $9.3 \mathrm{eV}$ and the self-trapped exciton emission at $2.8 \mathrm{eV}$, a $70 \%$ reduction; in amorphous 


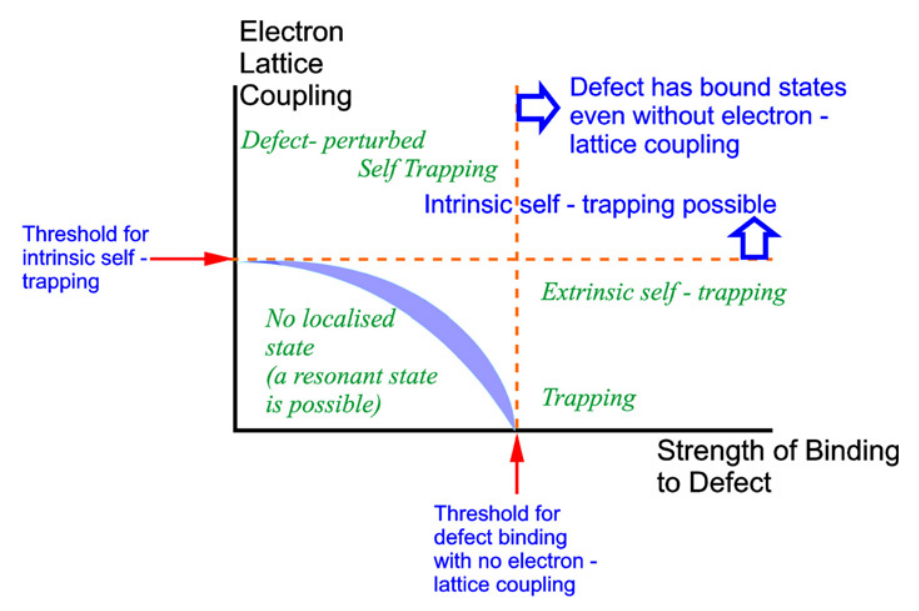

Figure 2. Trapping and self-trapping, indicating different regimes of behaviour (after Itoh and Stoneham 2001).

silica, there is a similar shift. In their table 8.2, Itoh and Stoneham (2001) list values for a number of systems.

Just as theory gives a very good description of the $V_{k}$ centre, so theory gives an excellent description of the self-trapped exciton (STX). The initial Hartree-Fock theory (Fisher et al 1990b, 1990a) gave very respectable descriptions of the optical energies, including the large Stokes shift, dichroism, spin resonance parameters, and transient volume change in $\alpha$-quartz. For $\alpha$-quartz, Van Ginhoven et al (2003) investigated the structure and properties of the selftrapped exciton using both density functional theory (DFT) and wavefunction-based (UHF, UMP2, CAS-SCF, and CCSD(T)) electronic structure methods, treating electron correlation and exchange to varying degrees of accuracy. They used cluster configurations extracted from supercell DFT calculations of the STX in $\alpha$-quartz, and found two luminescent STXs, together with a non-radiative state at a crossing of the singlet and triplet surfaces. One of the luminescent STXs corresponds to that found by Fisher et al $(1990 \mathrm{a}, 1990 \mathrm{~b})$ and, for $\beta$-crystobalite, by Shluger (1988) and Shluger and Stefanovich (1990). The second exciton structure may well be an artefact of the DFT calculations (Van Ginhoven et al 2003). The $\alpha$-quartz model of figure 1, with the excited electron and hole localized on the $\mathrm{Si}$ and $\mathrm{O}$ atoms of a broken $\mathrm{Si}-\mathrm{O}$ bond, has also been validated by Ismail-Beigi and Louie (2005), who combine a state-of-theart methods with a many-electron Green's function approach. They examine the mechanism of exciton self-trapping, and predict its structure and luminescence energy. They also found that the properties of $\alpha$-quartz favour exciton self-trapping since, even prior to self-trapping, the strong electron-hole attraction confines the electron close to the hole, so the kinetic energy cost of further localization of the exciton is relatively low, and is overcome by the substantial gain due to the lattice distortion.

\section{Trapping and self-trapping}

Trapping involves some local perturbation, such as a defect, an impurity, or a region of relatively high affinity in an amorphous solid. Trapping and self-trapping can, and often do, occur in combination (figure 2). As remarked above, Landau (1933) suggested there would be an energy barrier between self-trapped and free-electron states. Rashba (1957) then argued that a barrier is only possible in systems with more than two dimensions, and suggested the 
possible coexistence of free and self-trapped states. Mott (Mott and Stoneham 1977) came to the same view from simple arguments based on energy terms comprising a term related to the bandwidth, strain energy terms quadratic in the self-trapping displacement $Q$ and terms driving the self-trapping distortion that were linear in $Q$. Such a barrier is indeed observed for excitons in alkali iodides, but this is one of the very few cases where coexisting free and self-trapped excitons have been observed unambiguously. In most cases the barrier, if it exists, is hard to identify. In many systems, polaron self-trapping cannot be separated practically from trapping by weak potential of static lattice imperfections, such as intrinsic and extrinsic defects, or structural disorder in general. Shinozuka and Toyozawa (1979), Toyozawa (1990) proposed that the localizing effects of weak external potential and of electron-lattice coupling are additive, leading to localization even when neither separately is strong enough to give localization. This situation is often referred to as extrinsic self-trapping (Song and Williams 1993, Itoh and Stoneham 2001, figure 2 here) and is relevant for most systems considered below. The barrier can be eliminated in some situations in which there is localization of a trapped electron, e.g., a hole trapped by an $\mathrm{Mg}$ vacancy in $\mathrm{MgO}$ (the so-called $\mathrm{V}^{-}$centre; Mott and Stoneham 1977). Subsequent discussions of the self-trapping process are given in Shluger and Stoneham (1993). We shall return to these simple intrinsic oxide centres in oxides in section 5 .

\subsection{Polaron-like trapping by oxygen vacancy in $\mathrm{HfO}_{2}$}

Hafnium-based oxides have considerable potential for use in metal-oxide-semiconductor fieldeffect transistors (MOSFETs), primarily because their static dielectric permittivities $(\sim 22$ in $\left.\mathrm{HfO}_{2}\right)$ are much larger than that $(\sim 3.9)$ of the current dielectric, a-SiO${ }_{2}$. However, other properties must be considered (Stoneham et al 2005), and hafnia films tend to trap injected electrons, so accumulating negative charge that degrades performance. There is still debate on whether this is charge trapping by defects, or self-trapping. However, it is largely accepted that oxygen vacancies can enhance trapping significantly. There have been many studies of the electronic structure of these oxygen vacancies (Shluger et al 2003, Robertson 2006). What has become clear only recently is that so-called shallow electron trapping by the oxygen vacancy has a strong polaronic character (Gavartin et al 2006a, 2006b). We shall discuss carriers trapped at cation vacancies in cubic $\mathrm{MgO}$ in section 5.

Monoclinic $(\mathrm{m})-\mathrm{HfO}_{2}$ has a band gap $E_{\mathrm{g}} \sim 5.6-5.9 \mathrm{eV}$. Oxygen vacancies are common defects that are stable in several different charge states. Figure 3 shows charge densities for these charge states, calculated with a hybrid B3LYP density functional (Gavartin et al 2006a). Removing $\mathrm{O}^{2-}$ from the perfect crystal creates a doubly charged vacancy, $\mathrm{V}^{2+}$, with an unoccupied defect state in the band gap about $0.8 \mathrm{eV}$ below the conduction band minimum; in principle, this defect state might accommodate two electrons. Adding the first electron creates $\mathrm{a} \mathrm{V}^{+}$defect. Lattice polarization shifts the defect level by about $2 \mathrm{eV}$ to near the middle of the bandgap. The spin density for the $\mathrm{V}^{+}$state (figure 3(a)) is centred on the vacancy, just as in the familiar F-centres (e.g., Stoneham 1975), though the nearest-neighbour cations in m$\mathrm{HfO}_{2}$ also have a significant d-orbital contribution. Addition of a second electron to form $\mathrm{V}^{0}$ causes all ions to move back very close to their perfect lattice sites. The ground state of $\mathrm{V}^{0}$ is a spin singlet; no additional gap states are formed. Adding yet another electron (hence $\mathrm{V}^{-}$) shows the importance of the polarization of the lattice. Another localized gap state emerges from the conduction band minimum. This is an example of an extrinsic self-trapped polaron: the weak potential of the neutral vacancy aids localization of an extra electron to form the $\mathrm{V}^{-}$ state (figure $3(\mathrm{~b})$ ). The situation for $\mathrm{m}-\mathrm{HfO}_{2}$ is not at all like the $\mathrm{F}^{\prime}$-centre in alkali halides (see Stoneham 1975), for which electron correlation is more important than polaron effects. 


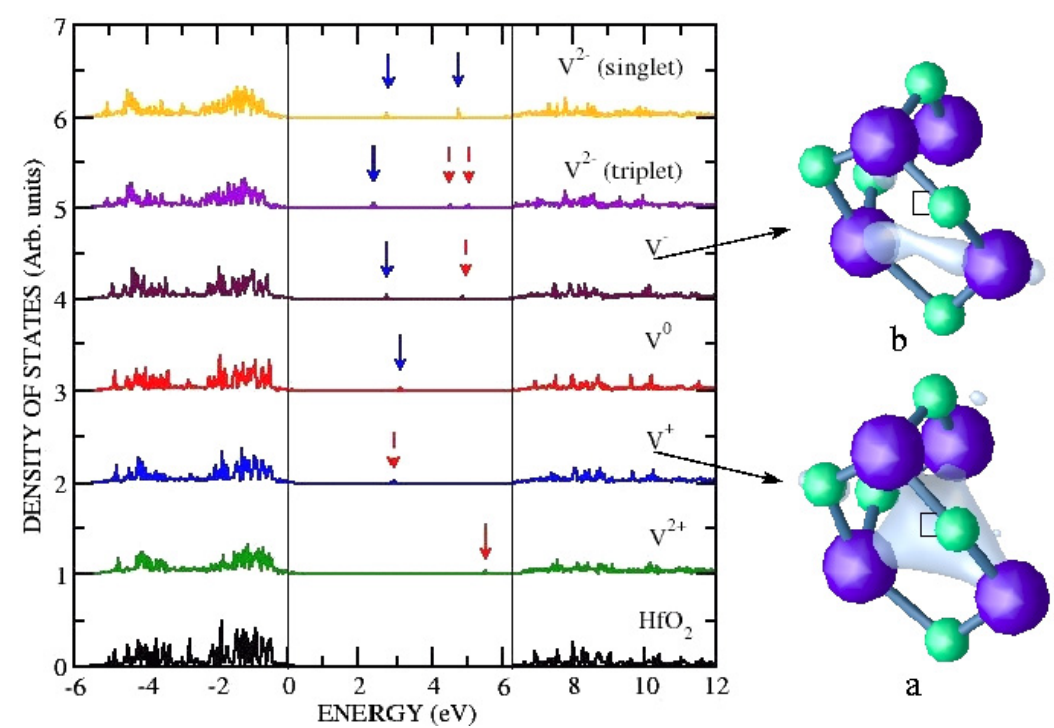

Figure 3. Electron density of states (majority spin) for the oxygen vacancy in $\mathrm{m}-\mathrm{HiO}_{2}$. The local levels associated with $\mathrm{V}^{+}$and $\mathrm{V}^{0}$ are seen as gap states, while the levels associated with $\mathrm{V}^{-}$and $\mathrm{V}^{2-}$ are seen as shallow band tail states near the conduction band minimum. The spin densities for $\mathrm{V}^{+}$(a) and $\mathrm{V}^{-}$(b) are shown on the right.

For $\mathrm{m}-\mathrm{HfO}_{2}$, a further electron can be bound, forming a $\mathrm{V}^{2-}$ state. The singlet state $\mathrm{V}^{2-}$ has a slightly lower energy than the triplet state. Recent recalculations of the free energies of the oxygen vacancy charge states (Xiong et al 2006) suggest the paramagnetic $\mathrm{V}^{+}$and $\mathrm{V}^{-}$states are thermodynamically unstable. Thus, if there were an unlimited supply of electrons, oxygen vacancies would be more stable as $\mathrm{V}^{2-}$, rather than $\mathrm{V}^{-}$.

The changes of vacancy charge state are accompanied by significant displacements of the nearest-neighbour Hf atoms and next shell of oxygen atoms, the relaxations being approximately symmetric about the vacancy site. The $\mathrm{Hf}$ neighbours of $\mathrm{V}^{-}$and $\mathrm{V}^{2-}$ displace towards the vacancy site by $4 \%$ and $8 \%$ of the $\mathrm{Hf}-\mathrm{O}$ distance; as one might guess from simple electrostatics arguments, these displacements are opposite to the small outward displacement for $\mathrm{V}^{+}$and the larger $\sim 10 \%$ outward displacement for $\mathrm{V}^{2+}$. For the $\mathrm{V}^{-}$and $\mathrm{V}^{2-}$, the oxygen second-neighbour displacements are substantially smaller and directed away from the vacancy. This electron density distribution, strong lattice relaxation and relatively shallow one-electron levels suggest that the trapping of the extra electrons at $\mathrm{V}^{-}$and $\mathrm{V}^{2-}$ is essentially polaronic in nature. The third and fourth electrons induce strong lattice polarization, which in turn creates the potential well for these electrons. Thus one might regard $\mathrm{V}^{2-}$ as an extrinsic bipolaron.

In some cases, it may be necessary to have a localizing imperfection to make observation possible, e.g., in the scanning tunnelling microscopy of Rønnow et al (2006; see also Mathur 2006). In the case of muons, the particle enters the solid at relatively high energy, and may trap an electron to form muonium. Since the initial energy will often be higher than any barrier to self-trapping, the barrier would be hard to identify. However, the imperfection may also catalyse self-trapping (Browne and Stoneham 1982).

\subsection{Static disorder: self-trapped excitons in amorphous $\mathrm{SiO}_{2}$}

Silicon dioxide shows both impurity- and disorder-related factors affecting the trapping and self-trapping of holes and excitons. Hole trapping in crystalline and amorphous $\mathrm{SiO}_{2}$ has 
been studied extensively (e.g., Hayes and Jenkin 1986, Sasajima and Tanimura 2002, Wang et al 2005). Doping of $\alpha$-quartz samples with $\mathrm{Ge}$ and $\mathrm{Al}$ impurities leads to hole trapping on an oxygen ion next to the impurity ion. The spectroscopic properties of these centres are summarized by Pacchioni et al (1989) and To et al (2005). However, neither holes nor electrons appear to self-trap in pure $\alpha$-quartz (Hayes and Jenkin 1986). Nevertheless, in amorphous $\mathrm{SiO}_{2}$, the electron and hole appear to self-trap, in contrast to quartz. The extensive electron spin resonance (ESR) and optical studies of Griscom $(1992,2006)$ show the existence of two types of trapped hole $(\mathrm{STH})$ in $\mathrm{x}$-irradiated bulk samples of amorphous $\mathrm{SiO}_{2}$. One $\left(\mathrm{STH}_{1}\right)$ has been assigned to a hole trapped on a single bridging oxygen, the other $\left(\mathrm{STH}_{2}\right)$ to a hole delocalized over two bridging oxygen atoms of the same $\mathrm{SiO}_{4}$ tetrahedron.

An extensive spectroscopic study of trapped holes in crystalline $\mathrm{SiO}_{2}$ has been carried out by To et al (2005). Recently, Kimmel et al (2006) employed a continuous random network model of amorphous silica in an embedded cluster method to study possible configurations and spectroscopic properties of trapped holes in amorphous silica. They used DFT with a modified hybrid density functional BLYP (Becke 1993, Lee et al 1988) and calibrated the Hartree-Fock exchange contribution (32.5\%) using known electronic and spectroscopic properties of holes trapped by Ge and Al impurities in $\alpha$-quartz. They then predicted the degree of localization and spectroscopic properties of holes at a set of sites in amorphous silica. These calculations showed that electrostatic potential fluctuations in the amorphous matrix provide precursor sites for the hole trapping. Good agreement was obtained between the experimental ESR and optical absorption energies attributed to $\mathrm{STH}_{1}$ and $\mathrm{STH}_{2}$ centres (Griscom 1992, 2006) and with the calculated parameters for the one- and two-centre holes trapped in amorphous silica. The optical transitions for $\mathrm{Ge}$ and $\mathrm{Al}$ hole centres in $\alpha$-quartz, for $\mathrm{STH}_{1}$ and $\mathrm{STH}_{2}$ in amorphous silica, and for the $\mathrm{V}^{-}$centre in $\mathrm{MgO}$ (Schirmer et al 1974) are all charge-transfer transitions, which can also be described as excitations from defect-induced resonant states in the valence band to unoccupied oxygen states.

Griscom has emphasized that $\mathrm{STH}_{1}$ and $\mathrm{STH}_{2}$ are not the only possible hole centres, and some 'mixed' configurations may be needed to understand the ESR spectra fully. This view is supported by the theory, which clearly shows the existence of two-centre holes with an intermediate degree of localization (Kimmel et al 2006). This work, which stresses the importance of precursor sites and the statistical analysis of defect configurations available in amorphous structures, has also been demonstrated by extensive studies of hole trapping by neutral oxygen vacancies in amorphous silica and formation of $E^{\prime}$ centres by Sushko et al (2005).

The Stokes shift of the self-trapped exciton luminescence is larger in fused silica than that of $\alpha$-quartz by $0.5-0.8 \mathrm{eV}$. Two factors may be involved. First, the energy of an exciton, whether self-trapped or not, will vary from site to site in an amorphous structure. Since both the core exciton and the normal exciton have relatively narrow absorption lines, the unrelaxed energies cannot vary much from site to site. The relaxation energies may vary, perhaps because of site-to-site variations in force constants, which make it easier to move the oxygen for certain local geometries (figure 4). Such site-to-site variations would affect both the stability (total energy) and the emission energy. A second factor arises because the exciton might diffuse for short distances to sites of lower energy, these also being sites of larger relaxation energy, where the luminescence will have a lower energy. The different behaviours of holes in $\alpha$-quartz and vitreous silica suggest relaxation energy variations of a few tenths of an eV are needed to explain the differences in exciton luminescence; these are not unreasonable. The importance of these issues has been recently demonstrated by Van Ginhoven et al (2006) who used DFT to model exciton configurations in amorphous silica, introducing triplet electron-hole excitations to study self-trapping (localization) and damage formation. Multiple 


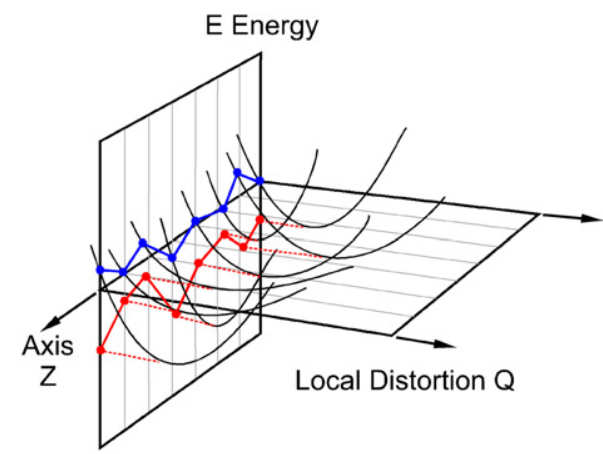

Figure 4. Schematic diagram (after Itoh and Stoneham 2001) showing the difference between simple fluctuations of potential from site to site, and variations in relaxation energy associated with self-trapping. Imagine the axis as a row of alternate $\mathrm{Si}$ and $\mathrm{O}$ atoms (-Si-O-Si-O-Si-) and that relaxation changes the $\mathrm{Si}-\mathrm{O}-\mathrm{Si}$ angle, as in self-trapping in quartz (figure 1). The figure shows variations in unrelaxed energies (blue) and in relaxation energies (red) through variations in forces and force constants. The exploitation of these lower relaxed state energies shifts the blue luminescence from $2.7 \mathrm{eV}$ in quartz to $2.1 \mathrm{eV}$ in a-SiO 2 .

self-trapped exciton states were found, and differentiated based on the luminescence energy, the localization and distribution of the excess spin density of the triplet state, and relevant structural data, including the presence or absence of broken bonds. Trapping is shown to be affected by the relaxation response of the silica network. The effects of the inherent disordered structures on exciton self-trapping were revealed by comparing results for quartz and amorphous silica. A key result is that the process of exciton trapping can lead directly to the formation of point defects, without thermal activation. The proposed mechanism includes a non-radiative decay from the excited to the ground state, followed by structure relaxation to a defect configuration in the ground state.

\subsection{Static disorder: electron polarons in disordered $\mathrm{HfO}_{2}$}

Hafnia does not easily amorphize unless alloyed with small amounts of some network former, like $\mathrm{Si}$ or Al. However, thin $(<5 \mathrm{~nm}) \mathrm{HfO}_{2}$ films on silicon appear to be amorphous and largely defect-free (Nguyen et al 2005). The best uniform amorphous hafnia films are observed to have densities in a range $90-98 \%$ of the crystalline value (Green et al 2002). Theory (Vanderbilt et al 2005, Ceresoli and Vanderbilt 2006) predicts amorphous $\mathrm{ZrO}_{2}$ and $\mathrm{HfO}_{2}$ at these densities; spontaneous crystallization occurs at higher densities. An important question is whether the atomic disorder in $\mathrm{HfO}_{2}$ implies potential fluctuations sufficient to trap electrons or holes. Gavartin et al (2006b) addressed the problem of electron self-trapping in disordered $\mathrm{HfO}_{2}$ with $95 \%$ of bulk density, exploiting simulated thermal annealing within a plane-wave density functional theory. Their approach used a projector-augmented-wave approach for the core electrons and the generalized gradient approximation for the spin density functional, as in VASP code (Kresse and Furthmüller 1996). Only those structures without localized gap states were investigated for self-trapping. One such structure is illustrated in figure 5(a). New calculations were then done with an extra one, two, three or four electrons, relaxing all atoms to accommodate the extra electrons. The resulting density of states for majority spin electrons is shown in figure 5(b). Conduction and valence band edge tails due to atomic disorder are evident even in the initial neutral structure. Adding electrons induces lattice polarization, allowing localization that can be seen from the appearance of gap localized states. The induced gap states 

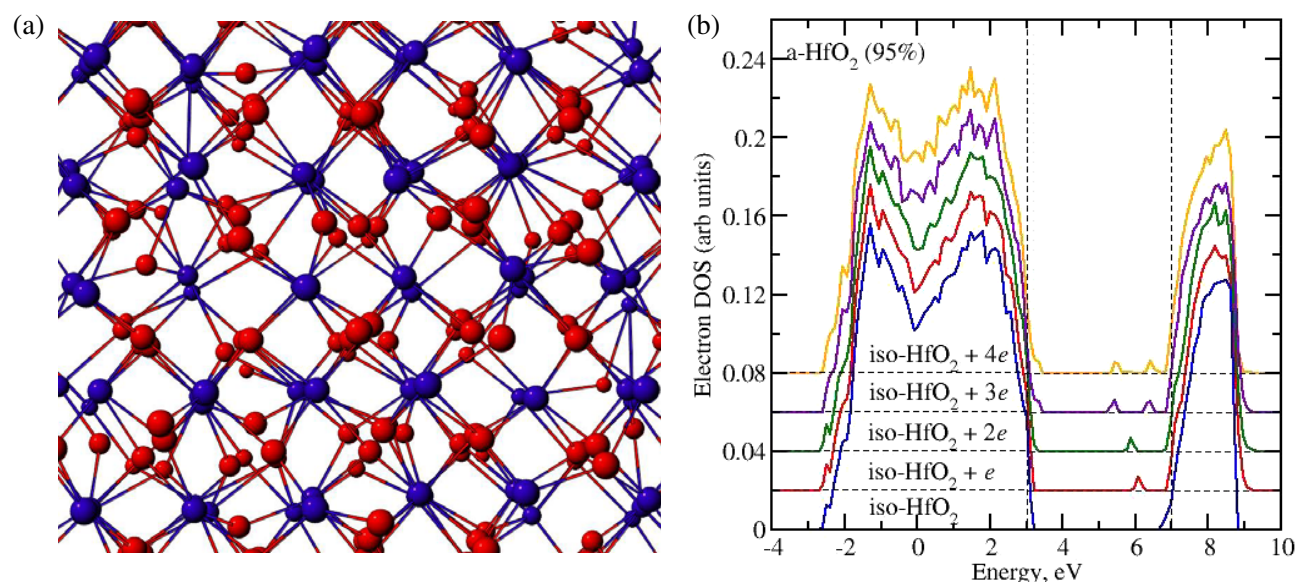

Figure 5. (a) A ball and stick model of disordered hafnia at $95 \%$ bulk density, containing $103 \mathrm{HfO}_{2}$ structural units. (b) The electron density of states of valence and conduction bands for the model of (a) for the neutral system (base line) and for fully relaxed systems containing one, two, three and four extra electrons. The corresponding densities of states are offset vertically for clarity. The vertical dashed lines indicate the band edge energies for perfect monoclinic $\mathrm{HfO}_{2}$.

have energies somewhat similar to those of the oxygen vacancies discussed earlier. Despite the clear signature in the electronic structure, it is hard to define vacancies in this topologically disordered system, with substantial fluctuations of atomic coordination numbers.

The calculations show that disordered $\mathrm{HfO}_{2}$ readily traps electrons, and the valence band tail suggests that holes may also be trapped by disorder. Interestingly, these conclusions can be reached even with the semi-local density functional used in this study. As noted above, this approximation is known to fail in many localization problems (Gavartin and Shluger 2001, Gavartin et al 2003), including polaron trapping by the oxygen vacancy in $\mathrm{m}-\mathrm{HfO}_{2}$, discussed earlier. Evidently, the electron-lattice interaction in disordered $\mathrm{HfO}_{2}$ is strong enough that even the local approximation is qualitatively correct, predicting a lattice distortion leading to deep defect-like states rather than the shallow states of a conventional polaron. However, in m- $\mathrm{HfO}_{2}$, the electron and hole small polarons, if they exist, form much shallower states. Clearly, a better description of the exchange interaction is needed to verify the situation theoretically. We know of no firm experimental evidence for intrinsic polarons in $\mathrm{HfO}_{2}$, though there is some evidence of exciton self-trapping in cubic $\mathrm{ZrO}_{2}$ and $\mathrm{HfO}_{2}$ films (Kirm et al 2005), i.e., similar materials.

\subsection{Scanning tunnelling microscope mapping of a trapped polaron}

Layered manganese oxide (manganite) is one member of the important class of complex ceramics exhibiting colossal magnetoresistance. Recent scanning tunnelling microscopy and spectroscopy (STM/STS) studies of this layered manganese oxide show what appears to be coherent in-plane motion (Rønnow et al 2006). Transport measurements show 'metallic' conduction within the $\mathrm{MnO}$ planes of $\mathrm{La}_{1.4} \mathrm{Sr}_{1.6} \mathrm{Mn}_{2} \mathrm{O}_{7}$ and insulating behaviour perpendicular to the planes between the Curie temperature and $250 \mathrm{~K}$. The transport data can be modelled as a quasiparticle that moves easily within the $\mathrm{MnO}$ planes, but which has to overcome a finite potential barrier to hop between neighbouring MnO bilayers. Rønnow et al proposed that the quasiparticle responsible for this behaviour is a polaron. Confirming this is not straightforward, since magnetic and Jahn-Teller terms are involved. In particular, is the polaron mobile within the plane as a large polaron or as a propagating small polaron regime? 


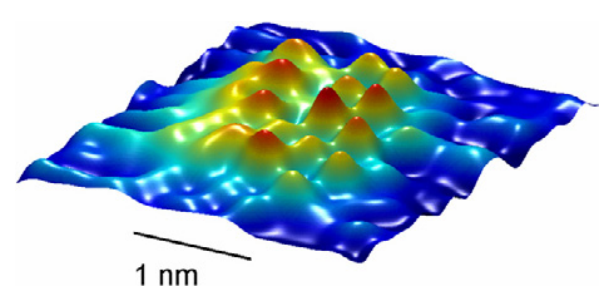

Figure 6. A three-dimensional rendering of the STM micrograph of a trapped small polaron in $\mathrm{La}_{1.4} \mathrm{Sr}_{1.6} \mathrm{Mn}_{2} \mathrm{O}_{7}$ (Rønnow et al 2006). The total vertical range amounts to $0.4 \AA$, and the lattice site spacing equals $3.86 \AA$, consistent with the $a b$-plane Mn unit cell.

The STM work of Rønnow et al (2006) shows there to be small regions where atomic resolution imaging can be achieved. These regions (figure 6) are interpreted as signatures of polarons localized by some sort of local imperfection. The size of the polaron is relatively large, perhaps $2 \mathrm{~nm}$ across, consistent with the polaron being spread over a small cluster of $\mathrm{MnO}_{6}$ octahedra. These atomically resolved islands have the same size as spin and lattice textures seen in neutron (Perring et al 1997) and x-ray scattering experiments (Campbell et al 2001, Vasiliu-Doloc et al 1999) which have been interpreted as polarons. In addition to these independent experimental observations for the same material system, tunnelling spectroscopy further supports the polaron interpretation of the STM micrographs. The zero-bias tunnelling conductance fits a temperature dependence of the form $T^{N} \exp (-\varepsilon / k T)$ with $\varepsilon \sim 0.1 \mathrm{eV}$. N is model dependent; with $N=3 / 2$, the value is $0.094 \mathrm{eV}$, the $\Delta / 2$ of Rønnow et al (2006); with $N=0$, the value is closer to $0.13 \mathrm{eV}$. Such values are within the range observed for small-polaron motion in transition metal oxides at these temperatures (Catlow et al (1977) and references therein). However, it is not entirely clear which process is characterized by this energy, since there are several polaron-related steps that might be involved. One energy is that needed to localize the mobile carriers in the MnO plane; such energies are strongly system dependent. A second energy corresponds to inter-plane hopping, which one might expect to be similar to the energies observed for reorientation of trapped carriers, typically a few tenths of an $\mathrm{eV}$. There is a clear need for atomistic simulation of possible models of this system, and these should also assess the suggestions by Bersuker and Goodenough (1997) of large low-symmetry polarons in high- $T_{\mathrm{c}}$ copper oxides.

\section{Polaron dynamics}

\subsection{Dynamics leading to self-trapping}

The phenomenological polaron theories are elegant and often physically transparent. Yet they rely on strong assumptions as to the dominant electron and phonon states and mechanisms for coupling: it is common to 'guess' the source of electron-phonon coupling and, hence, the polaron model. Full-scale electronic structure calculations make fewer assumptions, but can lose physical transparency and simplicity. It is challenging to establish links between the phenomenology and $a b$ initio theory, though it can be illuminating. These complementary approaches should be especially fruitful for self-trapping phenomena, especially in quantifying the critical electron-phonon coupling strength. Thus Gavartin (2001) and Gavartin and Shluger (2001) analysed the dynamical coupling of atomic fluctuations and valence and conduction band tail states in $\mathrm{MgO}$. These are the fluctuations essentially responsible for electron and hole self-trapping. Similar ideas have been exploited to study localization in amorphous 


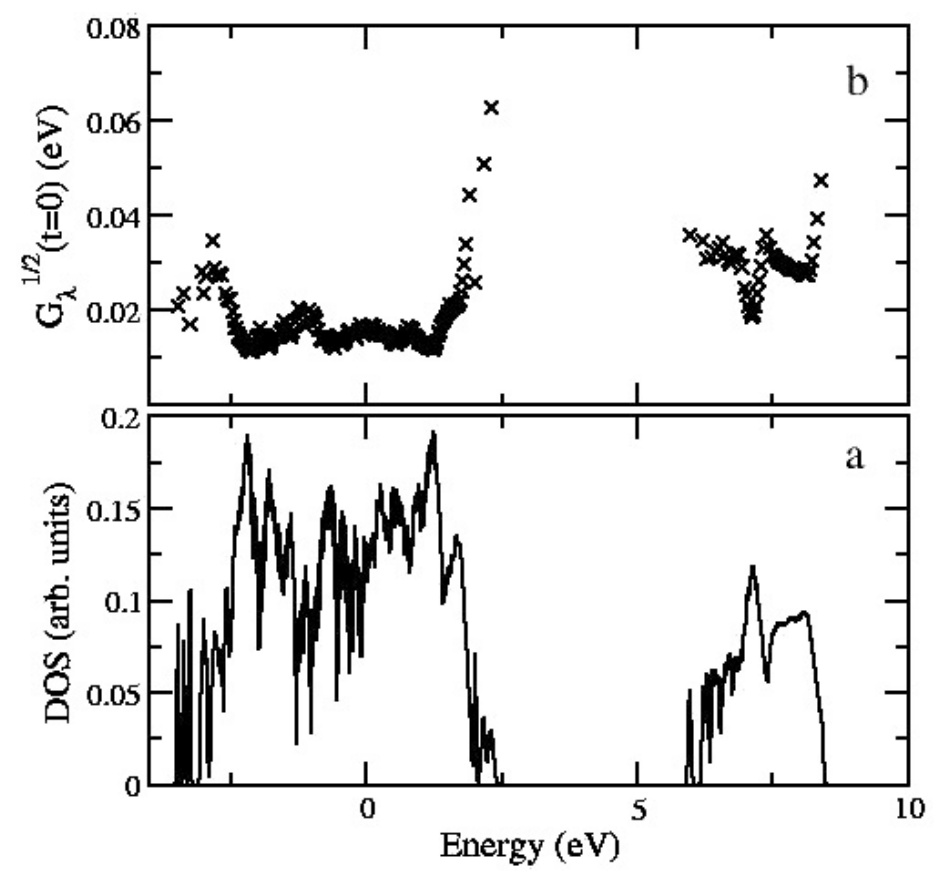

Figure 7. The lower figure shows the electronic density of states for a majority spin polaron in $\mathrm{m}-\mathrm{HfO}_{2}$. The upper figure shows the root mean square fluctuation, $\left\langle\lambda^{2}\right\rangle^{1 / 2}$, of the Kohn-Sham eigenstates as a function of their average energy $\langle\lambda\rangle$. Note the much larger fluctuations at the band edges (Gavartin and Shluger 2006).

semiconductors (Atta-Fynn et al 2004b, Abtew and Drabold 2004), who also suggested a universal link between the localization of states in band tails and their coupling strength to phonons (Atta-Fynn et al 2004a, Ludlam et al 2005). Calculations of the dynamical fluctuations of single-particle levels offer a powerful tool to study polaron trapping.

Gavartin and Shluger (2006) has made calculations for the polaron in $\mathrm{m}-\mathrm{HfO}_{2}$, giving the time evolution of self-consistent eigenvalues as they follow atomic displacements adiabatically within Born-Oppenheimer molecular dynamics, and using plane-wave density functional theory and the Kohn-Sham (KS) approximation. For each eigenvalue, $\lambda(t)$, the autocorrelation function $G_{\lambda}(t)=\langle\delta \lambda(\tau) \delta \lambda(\tau+t)\rangle$ can be obtained, and hence its Fourier transform, $G_{\lambda}(\omega)$, which is related to the frequency-dependent electron-phonon coupling strength. Here $\delta \lambda(t)$ denotes instantaneous deviation of the eigenvalue $\lambda(t)$ from its statistical average $\langle\lambda\rangle$, and $G_{\lambda}(t=0)$ is $\left\langle\delta \lambda^{2}\right\rangle$. It can be shown that $G_{\lambda}(\omega)$ is related to the polaron coupling strength to modes of frequency $\omega$. These calculations can be done both for perfect $\mathrm{m}-\mathrm{HfO}_{2}$ and for $\mathrm{m}-\mathrm{HfO}_{2}$ with an extra electron (polaron). The lower part of figure 7 shows the majority spin density of states for the system with a polaron, obtained as a superposition of Gaussian functions centred at $\langle\lambda\rangle$ with a dispersion $\left\langle\delta \lambda^{2}\right\rangle$; that for the perfect crystal (not shown) is very similar. The upper part of figure 7 shows the corresponding root mean square fluctuations $\left\langle\delta \lambda^{2}\right\rangle^{1 / 2}$ for all KohnSham eigenvalues, $\lambda$, as a function of $\langle\lambda\rangle$. Clearly, the Kohn-Sham energies near the band edges fluctuate far more than these in the middle of the bands. In particular, the root mean square fluctuation at the valence band maximum is about three times larger than average, reflecting their stronger interaction with phonons, in line with similar observations in semiconductors (Atta-Fynn et al 2004b, Abtew and Drabold 2004). 


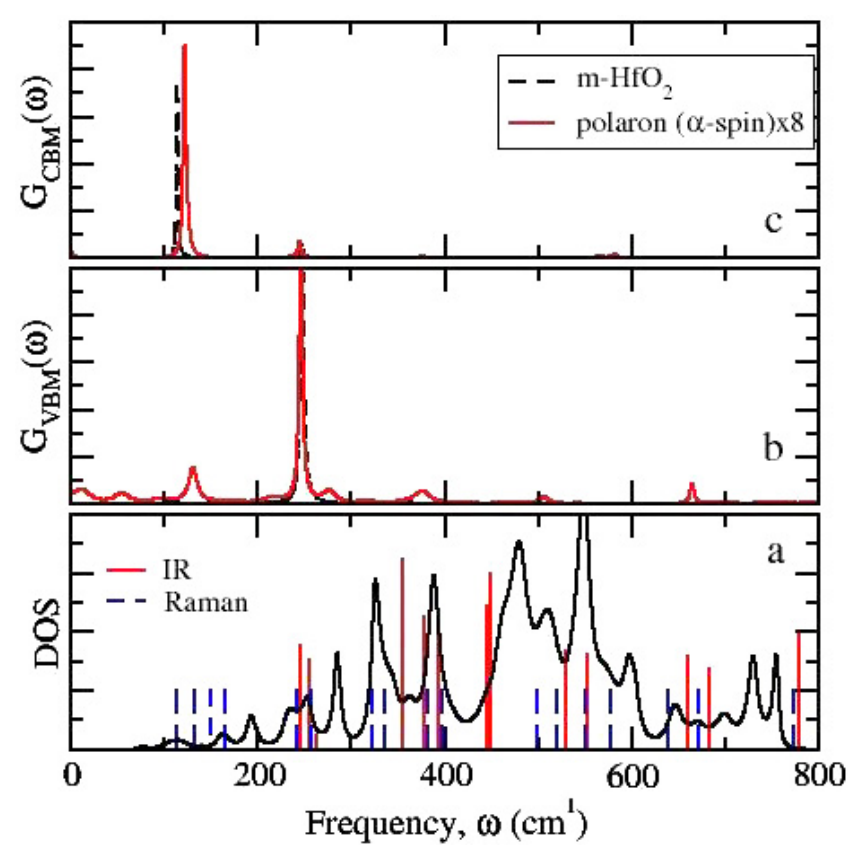

Figure 8. (a) Vibrational density of states for $\mathrm{m}-\mathrm{HfO}_{2}$ obtained from a Fourier transform of the velocity autocorrelation function. The vertical lines represent the infrared (solid) and Raman (dashed) active modes reported in literature. (b) Electron-phonon coupling strength to the valence band maximum Kohn-Sham eigenstate in the crystal with (solid) and without (dashed) a polaron. (c) Electron-phonon coupling strength to the conduction band minimum Kohn-Sham eigenstate in the crystal with (solid) and without (dashed) a polaron (Gavartin and Shluger 2006).

What do these results say about the electron-phonon coupling? Figure 8(a) shows the vibrational density of states from an ensemble average of the ionic velocity autocorrelation function. This figure also includes known experimental infrared and Raman frequencies (Arashi 1992, Nguyen et al 2005) and data from previous ab initio calculations (Zhao and Vanderbilt 2002, Rignanese 2005) for comparison. Figures 8(b) and (c) show $\left\langle\delta \lambda(\tau) G_{\lambda}(\omega)\right.$ $(\tau+t)\rangle$ for the valence band maximum and for the conduction band minimum. Evidently, the Kohn-Sham eigenvalue corresponding to the polaron state (at the bottom of the conduction band) is strongly coupled to phonons at $\sim 120 \mathrm{~cm}^{-1}$ and much less strongly to modes at $\sim 245 \mathrm{~cm}^{-1}$. The couplings to other phonons are far weaker and not seen at this scale. Coupling to the Kohn-Sham eigenvalue at the valence band maximum is less monochromatic, but the strongest contribution comes from a single phonon at $\sim 245 \mathrm{~cm}^{-1}$ and to a lesser extent from the band at $120 \mathrm{~cm}^{-1}$. Neither the conduction band maximum nor the valence band minimum eigenvalues show any significant coupling to the LO phonon at $\sim 770-780 \mathrm{~cm}^{-1}$ (Nguyen et al 2005). Interestingly, the presence of the electron at the bottom of the conduction band has little effect on the selective coupling: the single-peak character remains unaltered. However, the total electron-phonon coupling strength (the integral of $G(\omega)$ over $\omega$ ) is substantially smaller when a polaron is present (there is a factor 8 difference between the scales of figures $8(\mathrm{~b})$ and (c)).

The precise nature of the strongly coupled modes at 120 and $245 \mathrm{~cm}^{-1}$ has yet to be identified, though infrared active modes in this spectral region have been predicted for disordered $\mathrm{HfO}_{2}$ (Ceresoli and Vanderbilt 2006). More surprising is the very weak polaron 
coupling (if any) to the high-frequency LO phonon, especially in high- $k$ dielectrics. The results here strongly suggests that an electron at the bottom of the conduction band couples to much softer modes instead, and so to an elastic field, rather than polarization field. This makes the formation of self-trapped small-polaron states possible. The polaron state we observe is somewhat localized, albeit with character more consistent with weak band tail localization (Gavartin 2001, Gavartin and Shluger 2001, Atta-Fynn et al 2004a, 2004b) rather than a strongly localized self-trapped state, as seen earlier in disordered $\mathrm{HfO}_{2}$. The absence of selftrapping may be an artefact of the semi-local approximation (Gavartin et al 2003). So how might these results be reconciled with the standard view that charged carriers couple almost exclusively to the longitudinal optic modes? First, the special importance of the LO mode in Fröhlich's analysis stems from wavevector selection rules: the electron is deemed to have significant coupling only with those phonons with propagation direction collinear with $k$, the electron wavevector. The eigenvalue dynamics calculations make no assumption about the $\boldsymbol{k}$ dependence of coupling. Secondly, we analyse the coupling for the perfect lattice geometry so, in the case of carriers that self-trap, the natural interpretation is that it is these monochromatic coupled modes that initiate (nucleate) self-trapping. Whether or not the self-trapping occurs, and whether there is or is not a barrier to self-trapping, will depend on factors including coupling strengths to these modes (Gavartin and Shluger 2001). In systems that show only large-polaron behaviour, it may be that there are indeed other contributions to the polaron effective mass; experimentally, it is hard to separate the band mass from the polaron corrections. We note that Evans and Mills (1973) have analysed the interaction of slow electrons with the surface of a model dielectric, and comment on interactions with transverse optic modes as well as longitudinal optic modes.

Surfaces and interfaces lead to a range of phenomena relating to polarons. For example, the image interaction can help to stabilize the localization of a charge near to an interface with a medium of higher dielectric constant. This may be relevant for $\mathrm{SiO}_{2}$ near its interface with the higher dielectric constant Si. In quartz, carriers $(e, h)$ are close to self-trapping; in the amorphus oxide on silicon, self-trapping may be possible in some regions. It seems possible (Stoneham 1992) that the telegraph noise observed in $\mathrm{Si}$ near $\mathrm{SiO}_{2}$ (Kirton and Uren 1989) could involve electron transfers between the Si and small-polaron states in the oxide near the interface, without any specific structural or topological defect being involved.

\subsection{Coherent and incoherent motion}

Carrier motion can be either coherent or incoherent and, even in one host, both regimes of carrier motion can occur. Coherent motion can be of the characteristic large polaron form, as in $\mathrm{Si}$; mobilities fall with temperature as vibrational scatter increases. Mobilities remain above a few $\mathrm{cm}^{2} \mathrm{~V}^{-1} \mathrm{~s}^{-1}$ (the lower limit corresponding to mean free paths comparable to interatomic spacings). Incoherent processes may be thermally activated, with mobilities remaining below perhaps $0.1 \mathrm{~cm}^{2} \mathrm{~V}^{-1} \mathrm{~s}^{-1}$, a limit corresponding to about one jump every vibrational period. There can be distinct forms of coherent motion, perhaps the most interesting being the lowtemperature motion of self-trapped polarons when most of the modes relevant have very few or no phonons excited.

Most electron and hole small polarons $\left(V_{k}\right)$ seem to move by incoherent hopping at the temperatures at which they can be studied. Muons are examples of small polaron systems that show the lower-temperature coherent motion regime clearly, but the behaviour of hydrogen seems mainly in the incoherent regime, e.g., in hydrogen diffusion or reorientation, as in $\mathrm{Nb}$ : $(\mathrm{O}, \mathrm{H})$. The behaviour of muons is complicated by their short lifetime (a feature shared with self-trapped excitons) and by the fact that muons enter a solid with high energy, and presumably pass through a transient large-polaron coherent stage. 
Some studies of layered manganites appear to show coherent in-plane motion. Certainly the data of Rønnow et al (section 3.2 above) requires mobile carriers within the planes. This is important for cuprate systems, where there have been important suggestions that bipolaron systems underlie high- $T_{\mathrm{c}}$ behaviour (Alexandrov and Mott 1995, Müller 2006, Bussmann-Holder et al 2005, Bishop et al 2003). If small (bi)polarons are to explain oxide superconductivity, then in-plane coherent motion, as Rønnow et al (2006) see in the manganites, would seem essential.

\subsection{Electronic degeneracy: the Jahn-Teller effect and orbitons}

Both cuprate and manganite systems include transition metal ions with electronic degeneracy, these being so-called Jahn-Teller ions. The Jahn-Teller effect arises from electron-lattice coupling when there is electronic degeneracy or near-degeneracy. It is especially important that, for a given set of atomic positions, there are several accessible energy surfaces. Thus the Jahn-Teller effect is not simply another example of linear coupling, and a Jahn-Teller defect (irrespective of whether the Jahn-Teller effect is static or dynamic) is not simply a defect with rotational degrees of freedom. The extra degeneracy and different vibronic energy level structure leads to significantly different optical spectra (see especially Longuet-Higgins et al 1958) and to vibronic effects on matrix elements, such as the Ham effect (Ham 1965). These phenomena are reviewed in Stoneham (1975).

Isolated point defects can show either coherent (dynamic) behaviour or incoherent hopping between orientations. Thus the $\mathrm{MgO}: \mathrm{V}^{-}$centre shows incoherent reorientation, whereas $\mathrm{MgO}: \mathrm{Cu}^{2+}$ shows a dynamic Jahn-Teller effect which is essentially coherent rotation among the degenerate $x, y, z$ tetragonal distortions. The energy surface of the $\mathrm{Cu}^{2+}$ is double valued and, since the double-valued surface is not like that of a simple rotor, there are extra modes associated with the extra degrees of freedom. In particular, a crystal with many Jahn-Teller ions has collective excitations associated with movements of direction of the local JahnTeller distortion, somewhat similar to the motions of spin directions in spin waves (Sarfatt and Stoneham 1967). These motions now seem to be called orbitons (Allen and Perebeinos 2001) and there has been discussion of whether they have a role in the polaron-related behaviour of colossal magnetoresistance manganites.

\section{Optical and magnetic properties of sp oxides}

The understanding of many of the interesting properties of novel materials, like colossal magnetoresistance manganites, relies on analysis of optical and magnetic data. Often, the analysis adopts simple models for complex and subtle phenomena. We discuss in this section the results of previous detailed analyses of somewhat simpler systems for which rather full experimental data are available. This allows us to assess some of the approaches currently used.

\subsection{Localization of a trapped hole: the $\mathrm{MgO} \mathrm{V}^{-}$centre}

In an oxide like $\mathrm{MgO}$, free from transition metal ions, the $\mathrm{s}$ and $\mathrm{p}$ electrons determine the properties. Consider a cation vacancy: remove an $\mathrm{Mg}^{2+}$ ion and remove one electron (add one hole) to give a $\mathrm{V}^{-}$centre; with two charge-compensating holes, one would have a $\mathrm{V}^{0}$ centre. Both the $\mathrm{V}^{-}$and $\mathrm{V}^{0}$ centres can be observed in spin resonance; both have characteristic optical transitions; both have other observable properties as a check on any model. 
We know that the corresponding anion vacancy centres $\left(\mathrm{F}^{+}\right.$and $\left.\mathrm{F}^{0}\right)$ have cubic symmetry, with the electrons concentrated in the anion vacancy. A hole cannot localize in a vacancy, of course. But does the hole trapped at $\mathrm{V}^{-}$localize on one oxygen or spread over all six oxygen neighbours? There is a balance between the polarization energy (which favours localization) and tunnelling (which favours spreading). Spin resonance shows the hole localizes on just one of the oxygens, and there is incoherent hopping (slow at liquid helium temperatures) between equivalent configurations. It is relatively easy to estimate the polarization energy that encourages localization; in principle, accurate predictions of the tunnelling term are possible, but it is certainly sensible to identify an experimental value from optical data.

There are two main types of optical transition. The first type is an intra-site one at one oxygen; it is this transition energy that should be used in estimates of the $g$-factor. Predictions of energy and oscillator strength made by combining Hartree-Fock theory with shell model embedding (Norgett et al 1977) agree well with observation. The second class of transition is charge transfers (Schirmer et al 1974), in which the hole moves from one site to either a molecular orbital involving the four nearest oxygen neighbours or to the oxygen directly opposite on the other side of the vacancy. The optical transition energies for these charge transfers are dominated by polarization energies. Charge transfer provides the main optical transitions, and the two such transitions for $\mathrm{MgO}$ allow estimates of tunnelling and of polarization energies.

What emerges is that a combination of Hartree-Fock theory with embedding in a shell model works exceptionally well for spin resonance data, optical data, for the dipole moment (where there are some issues concerning effective field corrections that need resolution), reorientation energies, and data from trapping energetics. The same approach works equally well for optical charge transfer energies for related centres, e.g., $\mathrm{Na}$ or $\mathrm{K}$ at the $\mathrm{Mg}$ site, $\mathrm{Al}$ at the second-neighbour site, and second hole trapped to form a $\mathrm{V}^{0}$ centre. The major component in most of the calculated energies is obtained using the shell model, which separates ionic and electronic polarization in a systematic way that is general at the harmonic and dipole approximations. Rigid-ion models and single-frequency models fail badly. This is especially clear for the $\mathrm{V}^{-}$centre in ZnSe (Harding and Stoneham 1982) where the simple models that use a single vibrational frequency and do not separate ionic and electronic polarization fail in a significant way: the tunnelling parameters from the observed charge transfer optical spectra are such that the hole should not localize on a single Se, contrary to experiment. We note that many discussions of colossal magnetoresistance oxides adopt single-frequency models. Whilst these models can give insight, we emphasize that they can be inconsistent in important ways.

\section{2. $\mathrm{MgO}: V^{0}$ centres and possible ferromagnetism in simple oxides}

Measurements suggesting ferromagnetism in $\mathrm{HfO}_{2}$ (Coey et al 2005) have been controversial (Abraham et al 2005, Ramachandra Rao et al 1999, Hoa Hong et al 2006), though given some support from theory (Pemmajaru and Sanvito 2005). Ferromagnetic behaviour is seen in a number of transition metal doped oxides, e.g., $\mathrm{CeO}_{2}$ : Co (Tiwari et al 2006), $\mathrm{SnO}_{2}$ :Co and $\mathrm{SnO}_{2}: \mathrm{Fe}$ (Punnoose and Hays 2005), $\mathrm{TiO}_{2}: \mathrm{Co}$ (Jaffe et al 2005, Griffin et al 2005), usually in circumstances that need oxygen vacancy involvement. The precise mechanisms are unclear. These reports are tantalizing, since magnetism is unexpected in these robust and readily fabricated oxides and, if true, could be highly desirable. The $\mathrm{HfO}_{2}$ results suggest moments of one Bohr magneton for each few tens of molecular units, with useful magnetism at room temperature. Such behaviour could be the basis for new magnetic and spintronic devices. What mechanism might underlie the magnetism? 
There are natural parallels between these recent measurements and the earlier discussions of whether the $\mathrm{V}^{0}$ centre in II-VI oxides has a ground state that is a non-magnetic spin singlet or a magnetic spin triplet. The spin triplet is the puzzle. Start with a system of two electrons. If there are really only two electrons, the ground state will be a singlet (e.g., the He atom). However, if there are only two interesting electrons, the ground state can be a triplet (e.g., a $3 \mathrm{~d}^{2}$ system).

One suggestion of mechanism stems from analysis of cation vacancy centres in oxides (Stoneham (1975), Kollmar and Klein (1993) also cite a similar idea for organics due to McConnell (1967)). In the oxide of a divalent metal, the neutral cation vacancy can be considered as two holes trapped on oxygens adjacent to the site from which a divalent cation has been removed. Experiment shows that either the triplet state for the holes is the ground state ( $\mathrm{BeO}$ (Maffeo et al 1970), $\mathrm{Al}_{2} \mathrm{O}_{3}$ (Cox 1966, 1971, 1972)) or is close in energy to the groundstate singlet (MgO Wertz et al 1959, Rius and Cox 1974), $\mathrm{CaO}$ (Henderson and Tomlinson 1969, Abraham et al 1975, Henderson 2006); data are inconclusive for $\mathrm{ZnO}$ (Galland and Hervé 1970). Stoneham's suggestion was analysed in detail by Pathak et al (1976), combining shell model methods for the polarization and electric field aspects, and unrestricted HartreeFock theory for other aspects. The underlying idea is this. Consider a neutral $\mathrm{Mg}$ vacancy $\left(\mathrm{V}^{0}\right.$ centre) in $\mathrm{MgO}$, with two spins $1 / 2$ on opposite $\mathrm{O}^{-}$neighbours. All simple calculations (e.g., standard Hartree-Fock) will give $S=0$ as the ground state. However, one really has to include correlation through configuration admixture of the states that valence bond methods would include, i.e., $\mathrm{O}^{=} \mathrm{O}^{0}$ and $\mathrm{O}^{=} \mathrm{O}^{0}$ states are admixed into the dominant $\mathrm{O}^{-} \mathrm{O}^{-}$configuration. The admixed $S=1$ state (note that $\mathrm{O}^{0}$ has a triplet state lowest in energy, and the $\mathrm{O}^{=}$has a closed shell) is lower in energy than the admixed $S=0$ state. The CI correction will lower the triplet state in energy more than the singlet; as the correction from CI could be a few tens of meV, the competition between the two terms could lead to either the singlet or the triplet being lowest. In the case of $\mathrm{MgO}$, theory correctly predicts the $S=1$ state to be higher in energy by a few tens of meV, as observed. There is a very delicate balance of energies, however; recent careful density functional calculations for the same centre in $\mathrm{CaO}$ (Osorio-Guillén et al 2006) predict a very stable ferromagnetic state, contrary to experiment.

If this mechanism is correct, it could explain how isolated intrinsic defects in simple oxides might be ferromagnetic, even at room temperature. However, as (Osorio-Guillén et al 2006) have noted, genuine ferromagnetism needs connectivity between these defects, and the range of the inter-defect interactions seems too short to give this linkage. What about other defects? Is there a parallel explanation for anion vacancies? The answer is no for the simple sp oxides that have $\mathrm{F}^{+}$centres resembling the halide $\mathrm{F}$ centres, e.g., $\mathrm{MgO}, \mathrm{CaO}, \mathrm{BeO}, \mathrm{ZnO}, \mathrm{Al}_{2} \mathrm{O}_{3}$. For $\mathrm{MgO}$, the electrons localized by the anion vacancy in $\mathrm{F}^{+}$and $\mathrm{F}^{0}$ centres are primarily based on the site of the missing anion. For the two-electron $\mathrm{F}^{0}$ centre, there is a singlet state far below the triplet state. In essence, the tunnelling integral is substantial (several eV) and the polarization gain from localization reduced because it is the relatively unpolarizable $\mathrm{Mg}$ ions, not the polarizable oxygens, that are most affected. In principle, at least, electrons might localize on $\mathrm{Mg} \mathrm{p}$ states in $\mathrm{MgO}$ if the vacancy-centred states were eliminated implanting rare gas atoms. Anion vacancy complexes are known, e.g., the $\mathrm{F}_{t}$ centre (a [100] anion/cation/anion complex in $\mathrm{MgO}$ with two trapped electrons) and seem all to have $S=0$ antiferromagnetic coupling, but with low-lying $S=1$ states (Henderson 1966, Norgett 1971). Also seen is a [110] nearest-neighbour F-centre pair (Henderson and King 1967) with a triplet state occupied at $300 \mathrm{~K}$ but not at $4 \mathrm{~K}$.

The situation is different for those oxides with cations that can readily change valence, e.g., $\mathrm{TiO}_{2}$ or $\mathrm{HfO}_{2}$. One can see again how triplet states might arise. If one oxide ion $\mathrm{O}^{2-}$ is removed from perfect $\mathrm{TiO}_{2}$, the cations would normally have an empty d shell. Now add an electron. If the charge localizes, one of the closed-shell $\mathrm{Ti}^{4+}$ neighbours will become $\mathrm{Ti}^{3+}$. When a 
second electron is added, there are two possibilities. The second electron may localize on the same cation as the first, giving a $3 \mathrm{~d}^{2}$ ion that, by Hund's rules, will be in a spin triplet state. Alternatively, the second electron could localize on a different cation neighbour to the oxygen vacancy, probably on the opposite side of the $\mathrm{O}$ vacancy to minimize Coulomb repulsion. If so, then, just as for the $\mathrm{V}^{0}$ centres in $\mathrm{MgO}$, there can be configuration admixture, lowering the $S=1$ state relative to the $S=0$ Hartree-Fock ground state because of the Hund's rule lowering of the excited ionic triplet state relative to the corresponding singlet.

Overall, it appears that when the delicate balance between singlet and triplet states favours the triplet state, it requires polaronic localization of charge in one form or another. The coupling will often provide populations of spin triplet systems even at room temperature, but the concentrations and connectivity put severe limits on useful magnetic behaviour.

\section{Conclusions}

'Polaronic' behaviour encompasses those situations when strong electron phonon coupling leads to a qualitative change of behaviour. Often symmetry appears to be broken. Free holes may localize and hop incoherently, rather than propagate over many lattice spacings. Such selftrapping is a very common phenomenon. Trapped holes may localize on just one of several symmetry-equivalent ions neighbouring a defect, hopping incoherently between these sites. Such cases illustrate the important phenomenon of charge localization. In many interesting cases, trapping —involving an impurity or defect-and self-trapping operate together. Systems are observed over the whole range from purely polaronic effects (involving no defect, no impurity, no disorder) to ones for which defect properties are simply modified by the electronphonon coupling. In real systems, standard models provide only a limited framework. Many common assumptions developed for weakly coupled systems need reconsideration and generalization for strongly coupled systems. There is a further complication, in that the word 'polaron' is used very widely, but in several senses, so encompassing a range of behaviours (see, e.g., Lindgard and Stoneham 2003), especially for organic systems. Electronic excited states of self-trapped systems add new features of interest. Electronic excitation underlies material modification, where energy localization (as for self-trapped excitons) is a critical issue. The existence of alternate excited states (intra-site versus charge transfer, for instance) and the possibility of defect magnetism and Jahn-Teller excitations promise significant new physics. Material modification is only one example of the technological implications of polaron science. Polaron concepts remain a powerful and central idea in the science and technology of non-metals, and their role in quantum behaviour and in spintronics shows signs of proving important.

\section{Acknowledgments}

This paper is based on an invited talk at the symposium 'Mott's Physics in Nanowires and Quantum Dots', supported by the European Science Foundation. Nevill Mott was a consultant to Harwell from 1947 until about 1993, and the discussions he had with one of us (AMS) stimulated some of the work described in this paper.

\section{References}

Abraham D W, Frank M M and Guha S 2005 Appl. Phys. Lett. 87252502

Abraham M M, Chen Y, Boatner L A and Reynolds R W 1975 Solid State Commun. 161209

Abtew T A and Drabold D A 2004 J. Phys.: Condens. Matter 16 S5289-96

Alexandrov A S and Mott N F 1995 Polarons and Bipolarons (Singapore: World Scientific) 
Allen P B and Perebeinos V 2001 Nature 410155

Arashi H 1992 J. Am. Chem. Soc. 75844

Atta-Fynn R, Biswas P and Drabold D A 2004a Phys. Rev. B 69254204

Atta-Fynn R, Biswas P, Ordejón P and Drabold D A 2004b Phys. Rev. B 69085207

Becke A D 1993 J. Chem. Phys. 985548

Bersuker G I and Goodenough J B 1997 Physica C 274267

Bishop A R, Mihailovic D and Mustre de León 2003 J. Phys.: Condens. Matter 15 L169

Browne A M and Stoneham A M 1982 J. Phys. C: Solid State Phys. 15 2709-20

Bussmann-Holder A, Keller H and Müller K A 2005 Evidences for polaron formation in cuprates Structure and Bonding vol 114 (Berlin: Springer)

Cade P, Stoneham A M and Tasker P W 1984 Phys. Rev. B 30 4621-39

Campbell B J et al 2001 Phys. Rev. B 65014427

Catlow C R A, Mackrodt W C, Norgett M J and Stoneham A M 1977 Phil. Mag. 35177

Ceresoli D and Vanderbilt D 2006 Phys. Rev. B 74125108

Coey J M D, Venkatesan M, Stamenov P, Fitzgerald C B and Dorneles L S 2005 Phys. Rev. B 72024450

Cox R T 1966 Proc. XIV Colloque Ampere (Ljubljana) ed R Blinc (Amsterdam: North-Holland) p 279

Cox R T 1971 Solid State Commun. 91989

Cox R T 1972 Thesis University of Grenoble 306-7

Devreese J 1963 Bull. Soc. Belge Phys. Ser. III (4) 259-62

Emin D and Holstein T 1976 Phys. Rev. Lett. 36323

Evans E and Mills D L 1973 Phys. Rev. B 84004

Feynman R 1955 Phys. Rev. 97660

Fisher A J, Hayes W and Stoneham A M 1990a J. Phys.: Condens. Matter 2 6707-20

Fisher A J, Stoneham A M and Hayes W 1990b Phys. Rev. Lett. 64 2667-70

Fröhlich H 1962 Polarons and Excitons ed C G Kuper and G D Whitfield (Edinburgh: Oliver and Boyd)

Galland D and Hervé A 1970 Phys. Lett. A 33 1-2

Gavartin J L 2001 J. Phys.: Condens. Matter 1310873

Gavartin J L, Muñoz Ramo D and Shluger A L 2006a Appl. Phys. Lett. 89082908

Gavartin J L, Muñoz-Ramo D, Shluger A L and Bersuker G I 2006b Physics and Technology of High-k Dielectrics vol 4, ed S Kar, M Houssa; ECS Trans. 3277

Gavartin J L and Shluger A L 2001 Phys. Rev. B 64245111

Gavartin J L and Shluger A L 2006 Phys. Status Solidi C 3 at press

Gavartin J L, Sushko P V and Shluger A L 2003 Phys. Rev. B 67035108

Green M L, Ho M-Y, Busch B, Wilk G D, Sorsch T, Gonard T, Brijs B, Vandervost W, Räisänen P I, Muller D, Bude M and Grazul J 2002 J. Appl. Phys. 927168

Griffin K A, Pakhomov A B, Wang C M, Heald S M and Krishnan K M 2005 J. Appl. Phys. 97100318

Griscom D L 1992 J. Non-Cryst. Solids 149137

Griscom D L 2006 J. Non-Cryst. Solids 3522601

Ham F S 1965 Phys. Rev. A 1381727

Hampton R N, Saunders G A, Harding J H and Stoneham A M 1987 J. Nucl. Mater. 15017

Harding J H and Stoneham A M 1982 J. Phys. C: Solid State Phys. 15 4649-59

Hayes W and Jenkin T J L 1986 J. Phys. C: Solid State Phys. 196211

Hayes W and Stoneham A M 1985 Defects and Defect Processes in Non-Metallic Crystals (New York: Wiley)

Henderson B 1966 Br. J. Appl. Phys. 17851

Henderson B 2006 private communication

Henderson B and King R D 1967 J. Physique Coll. C4 (Suppl to no 8-9) 75

Henderson B and Tomlinson A C 1969 J. Phys. Chem. Solids 301801

Hoa Hong N, Poirot Ne and Sakai J 2006 Appl. Phys. Lett. 89042503

Holstein T 1959 Ann. Phys. NY 8 325-89

Huang K and Rhys A 1950 Proc. R. Soc. A 204406

Ismail-Beigi S and Louie S G 2005 Phys. Rev. Lett. 95156401

Itoh N and Stoneham A M 2001 Materials Modification by Electronic Excitation (Cambridge: Cambridge University Press)

Jaffe J E, Droubay T C and Chambers S A 2005 J. Appl. Phys. 97073908

Kanzig W 1955 Phys. Rev. 991890

Kimmel A, Sushko P V and Shluger A L 2006 J. Non-Cryst. Solids at press

Kirm M, Aarik J, Jurgens M and Sildos I 2005 Nucl. Instrum. Methods Phys. Res. A 537 251-5

Kirton M J and Uren M J 1989 Adv. Phys. 38367 
Kollmar C and Klein O 1993 Acc. Chem. Res. 26 259-65

Kresse G and Furthmüller J 1996 Comput. Mater. Sci. 615

Laegsgaard J and Stokbro K 2001 Phys. Rev. Lett. 862834

Landau L D 1933 Phys. Zeit Sowjetunion 3664

Lee C, Yang W and Parr R G 1988 Phys. Rev. B 37785

Lindgard P-A and Stoneham A M 2003 J. Phys.: Condens. Matter 15 V5-9

Longuet-Higgins H C, Öpik U, Pryce M H L and Sack R A 1958 Proc. R. Soc. A 2441

Ludlam J J, Taraskin S N, Elliott S R and Drabold D A 2005 J. Phys.: Condens. Matter 17 L321-7

Maffeo B, Hervé A and Cox R T 1970 Solid State Commun. 8 2169-72

Mathur N 2006 Nat. Phys. 2307

McConnell H M 1967 Proc. Robert A Welch Found Conf. Chem. Res. vol 11, p 144

Mott N F and Littleton M J 1938 Trans. Faraday Soc. 34 485-99

Mott N F and Stoneham A M 1977 J. Phys. C: Solid State Phys. $103391-8$

Müller K A 2006 From single- to bi-polarons with Jahn-Teller character and metallic cluster stripes in hole-doped cuprates Treatise on High Temperature Superconductivity ed J R Schrieffer at press

Nguyen N V, Davydov A V, Chandler-Horowitz D and Frank M M 2005 Appl. Phys. Lett. 87192903

Norgett M J 1971 J. Phys. C: Solid State Phys. 41289

Norgett M J, Pathak A P and Stoneham A M 1977 J. Phys. C: Solid State Phys. 10 555-65

Norgett M J and Stoneham A M 1973a J. Phys. C: Solid State Phys. $6229-37$

Norgett M J and Stoneham A M 1973b J. Phys. C: Solid State Phys. 6 238-46

Osorio-Guillén J, Lany S, Barabash S V and Zunger A 2006 Phys. Rev. Lett. 96107203

Pacchioni G, Frigoli F, Ricci D and Weil J A 2001 Phys. Rev. B 63054102

Pacchioni G, Skuja L and Griscom G 1989 Phys. Rev. B 406

Pathak A P, Bartram R H and Stoneham A M 1976 J. Phys. C: Solid State Phys. 9 73-80

Pekar S 1951 Issledovaniya po Elektronnoi Teorii Kristallov (Moscow: GITTL)

Pemmajaru C D and Sanvito S 2005 Phys. Rev. Lett. 94217205

Perring T G, Aeppli G, Moritomo Y and Tokura Y 1997 Phys. Rev. Lett. 783197

Puchin V E, Shluger A L, Tanimura K and Itoh N 1993 Phys. Rev. B 47 6226-40

Punnoose A and Hays J 2005 J. Appl. Phys. 97100321

Ramachandra Rao M S, Kundaliya D C, Ogale S B, Fu L F, Welz S J, Browning N D, Zaitsev V, Varughese B, Cardoso A, Curtin A, Dhar S, Shinde S R and Vasiliu-Doloc T 1999 Phys. Rev. Lett. 834393

Rashba E 1957 Opt. Spektrosk. 275

Rignanese G-M 2005 J. Phys.: Condens. Matter 17 R357

Rius G and Cox R 1974 private communication

Robertson J 2006 Rep. Prog. Phys. 69327

Rønnow H M, Renner Ch, Aeppli G, Kimura T and Tokura Y 2006 Nature 4401025

Sarfatt J and Stoneham A M 1967 Proc. Phys. Soc. 91 214-21

Sasajima Y and Tanimura K 2002 Phys. Rev. B 68014204

Schirmer O F, Koidl P and Reik H G 1974 Phys. Status Solidi b 62385

Shinozuka Y and Toyozawa Y 1979 J. Phys. Soc. Japan 46505

Shluger A L 1988 J. Phys. C: Solid State Phys. 21 L431

Shluger A L, Foster A S, Gavartin J L and Sushko P V 2003 Nano and Giga Challenges in Microelectronics ed J Greer, A Korkin and J Labanowski (Berlin: Elsevier) pp 151-222

Shluger A L, Kantorovich L N, Heifets E N, Shidlovskaya E K and Grimes R W 1992 J. Phys.: Condens. Matter 4 7417-28

Shluger A L, Puchin V E, Suzuki T, Tanimura K and Itoh N 1995 Phys. Rev. B 52 4017-28

Shluger A L and Stefanovich E V 1990 Phys. Rev. B 42 9664-73

Shluger A L and Stoneham A M 1993 J. Phys.: Condens. Matter 1 3049-86

Song K S and Williams R T 1993 Self-Trapped Excitons (Berlin: Springer)

Stoneham A M 1975 Theory of Defects in Solids (Oxford: Oxford University Press)

Stoneham A M 1989 J. Chem. Soc. Faraday II 85 505-16

Stoneham A M 1992 Insulating Films on Semiconductors 1991 ed W Eccleston and M Uren (Bristol: Hilger) pp 19-32

Stoneham A M, Gavartin J L and Shluger A L 2005 J. Phys.: Condens. Matter 17 S2027

Stoneham A M and Sangster M J L 1980 Phil. Mag. B 43 609-19

Sushko P V, Mukhopadhyay S, Mysovsky A, Sulimov V B, Taga A and Shluger A L 2005 J. Phys.: Condens. Matter $17 \mathrm{~S} 2115$

Tasker P W and Stoneham A M 1977 J. Phys. Chem. Solids 38 1185-9 
Tiwari A, Bhosle V M, Ramachandran S, Sudhakar N, Narayan J, Budak S and Gupta A 2006 Appl. Phys. Lett. 88142511

To J, Sokol A and French S A 2005 J. Chem. Phys. 12214470

Toyozawa Y 1962 Polarons and Excitons ed C G Kuper and G D Whitfield (Edinburgh: Oliver and Boyd)

Toyozawa Y 1990 Rev. Solid State Sci. 4133

Vanderbilt D, Zhao X and Ceresoli D 2005 Thin Solid Films 486125

Van Ginhoven R M, Jonsson H and Corrales L R 2006 J. Non-Cryst. Solids at press

Van Ginhoven R M, Jonsson H, Peterson K A, Dupuis M and Corrales L R 2003 J. Chem. Phys. 1186582

Vasiliu-Doloc L, Rosenkranz S, Osborn R, Sinha S K, Lynn J W, Mesot J, Seeck O H, Preosti G, Fedro A J and Mitchell J F 1999 Phys. Rev. Lett. 834393

Wang R P, Saito K and Ikushima A J 2005 J. Non-Cryst. Solids 3511569

Wertz J E, Auzins P, Griffiths J H E and Orton J W 1959 Discuss. Faraday Soc. 28136

Xiong K, Robertson J and Clark S J 2005 Phys. Status Solidi b 2432071

Xiong K, Robertson J and Clark S J 2006 Appl. Phys. Lett. 871

Yamashita J and Kurosawa T 1958 J. Phys. Chem. Solids $534-43$

Zhao X and Vanderbilt D 2002 Phys. Rev. B 65233106 